\title{
Epidermodysplasia verruciformis: three case reports and a brief review
}

\author{
Sharma Shruti ${ }^{1}$, Fouzia Siraj ${ }^{1 凶}$, Avninder Singh $^{1}$, V Ramesh $^{2}$
}

\begin{abstract}
Epidermodyplasia verruciformis (EV) is a rare heritable disease that is characterized by an increased susceptibility to infection with specific human papillomavirus (HPV) types due to a defect in cell-mediated immune response to HPV infection. Widespread infection with HPV is responsible for the development of wart-like lesions and pityriasis versicolor-like spots. These individuals have a lifelong risk of developing cutaneous malignancies, especially Bowen's disease and squamous cell carcinoma, mainly in sun-exposed parts. Being the first disease to correlate cancer and viral infection, EV serves as the cornerstone of the understanding of viral oncogenesis. We report three cases of EV, of which one patient subsequently developed Bowen's disease. The patients had multiple hypopigmented papules and plaques of varying sizes that started erupting in childhood and were mainly distributed over sun-exposed parts of the body. Histopathology of the skin biopsies was consistent with EV. One of these patients had started developing an ulcerated plaque over the left clavicle 2 years earlier. A biopsy from the clavicular region also showed histopathological features of Bowen's disease arising in EV. Hence, this clinical review discusses three cases of EV presenting in different age groups with detailed histopathological findings typical for EV.
\end{abstract}

Keywords: epidermodysplasia verruciformis, human papillomavirus, malignancy

Received: 17 January 2017 | Returned for modification: 12 March 2017 | Accepted: 24 May 2017

\section{Introduction}

Epidermodysplasia verruciformis (EV) is an uncommon, lifelong, autosomal recessive genodermatosis that involves the immune system (1). Clinically characterized by a spectrum of wart-like verrucous lesions and pityriasis versicolor-like spots, it starts in early childhood on the face, dorsum of the hands, and legs (2). These lesions are caused by infection with specific human papillomavirus (HPV) types that usually do not cause any clinical symptoms in the general population, whereas in patients with EV some HPV types can cause the development of flat wart-like lesions, which may progress to cancer (3). These include seborrheic keratosis, actinic keratosis, and cutaneous malignancies such as Bowen's disease and squamous cell carcinoma (SCC) after sun exposure of over 20 to 30 years (2). More than $30 \mathrm{EV}$-associated HPV types have been identified, including HPV5, HPV8, HPV12, HPV14, HPV15, HPV17, HPV19-HPV25, HPV36, HPV38, HPV47, and HPV50. Although these HPV types can also be detected in the general population, they are only pathogenic in EV patients, with HPV 5 and HPV8 known for their malignant potential (3).

This clinical review discusses three cases of EV presenting in different age groups with detailed histopathological findings typical for EV.

\section{Case reports}

Case 1: A 14-year-old boy residing in a rural area in India presented to the dermatology clinic with complaints of multiple, nonitchy hypopigmented papules and plaques that were distributed over the forehead, neck, trunk, and abdomen. They ranged from 0.5 to $2 \mathrm{~cm}$ in diameter and first erupted at the age of 6 months. The lesions gradually progressed in size and number. The patient had never consulted a doctor before and hence had not had any treatment for the skin lesions. There was no family history of skin disease. Punch skin biopsies were taken from the lesions on the trunk and abdomen.

Case 2: A 15-year-old boy presented to the dermatology outpatient department with multiple hypopigmented non-itchy papules ranging in size from 0.2 to $0.5 \mathrm{~cm}$ over the trunk and both hands that started 7 years previously. Similar lesions or a history of skin disease was not present in any family members. This was the first time the patient had consulted a doctor for his skin ailment. There was no other associated disease. Punch skin biopsy was taken from the hand and trunk.

In Cases 1 and 2, the boys attended school in rural areas and had to travel long distances in the sun every day.

Case 3: A 42-year-old woman presented with a $2 \mathrm{~cm}$ ulcerated, crusted plaque over the left clavicular region that had erupted 2 years earlier. Initially the plaque was flat, but it gradually developed a crust and during the previous month it had started ulcerating. Numerous flat, wart-like lesions varying in size from 0.5 to 1 $\mathrm{cm}$ were also present on the back, abdomen, and upper and lower limbs (Fig. 1). These flat, wart-like lesions had started developing over the body from age 12, gradually increasing in number and size. Excisional skin biopsy was performed on the ulcerated lesion over the clavicular region and a punch biopsy was taken from a lesion on the back. The patient did not have any other clinical disease or a family history of similar lesions. She was a worker on a farm and thus had a history of regular sun exposure.

On physical examination, the scalp, nails, genital area, and other mucous membranes were unremarkable in all three patients. They were immunocompetent and had no history of organ transplantation, Hodgkin's disease, systemic lupus erythematosus, or HIV infection.

Two punch skin biopsies from different sites were performed in all patients, and all of them showed similar histopathological findings. There were hyperkeratosis, acanthosis, and enlarged vacuolated keratinocytes with abundant bluish-gray cytoplasm beneath a variably thickened granular layer (Fig. 2). The clinical presentation was characteristic in all the cases and the histo- 
pathological features were diagnostic of epidermodysplasia verruciformis. An excisional biopsy from the clavicular region of the 42-year-old female patient (Case 3) also showed a focal fullthickness keratinocyte dysmaturation and dysplasia. There were variable nuclear pleomorphisms and numerous mitotic figures. Koilocytic changes were noted in superficial keratinocytes. Due to the absence of invasion, the histopathology was suggestive of Bowen's disease (squamous carcinoma in situ, CIS) arising in a lesion of EV (Fig. 3a, b).

At 1 and 2 years follow-up, no suspicious lesions developed in the boys in Cases 1 and 2. There was no recurrence or eruption of new lesions of Bowen's disease in Case 3.

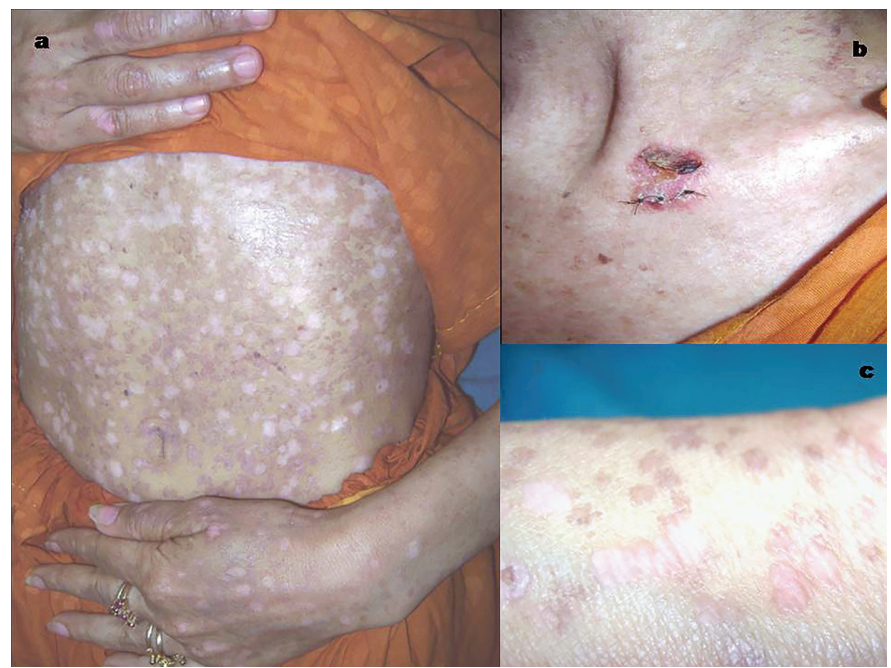

Figure 1 | (a, c) Multiple hypopigmented lesions over the abdomen and hands; (b) an ulcerated lesion over the clavicle.

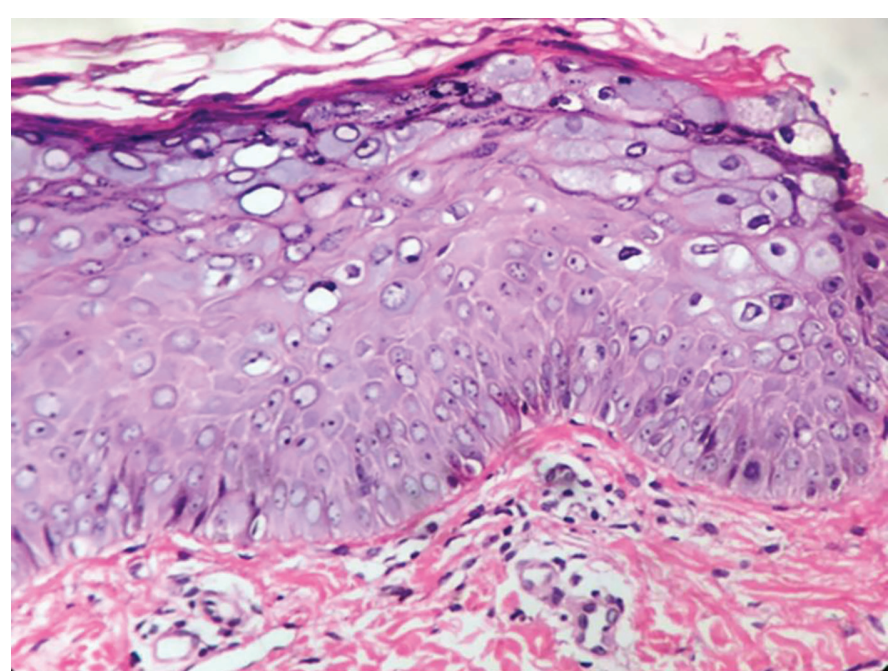

Figure 2 | Epidermis with acanthosis, hypergranulosis, and large cells with abundant blue-grey cytoplasm (H\&E, 20x).

\section{Discussion}

Epidermodysplasia verruciformis is a lifelong disorder of cell-mediated immunity (CMI) with no sex, racial, or geographic predisposition. It is a rare, autosomal recessive genodermatosis known for an increased risk of skin carcinomas (4). Although most cases of EV are autosomal recessive, cross-linked inheritance and sporadic mutations have also been documented in the literature. The familial form of EV is more common, but none of our cases elicited any family history of the disease and hence we assume that they had a sporadic appearance. Acquired forms of the disease are usually seen in immunodeficient patients such as after renal 60
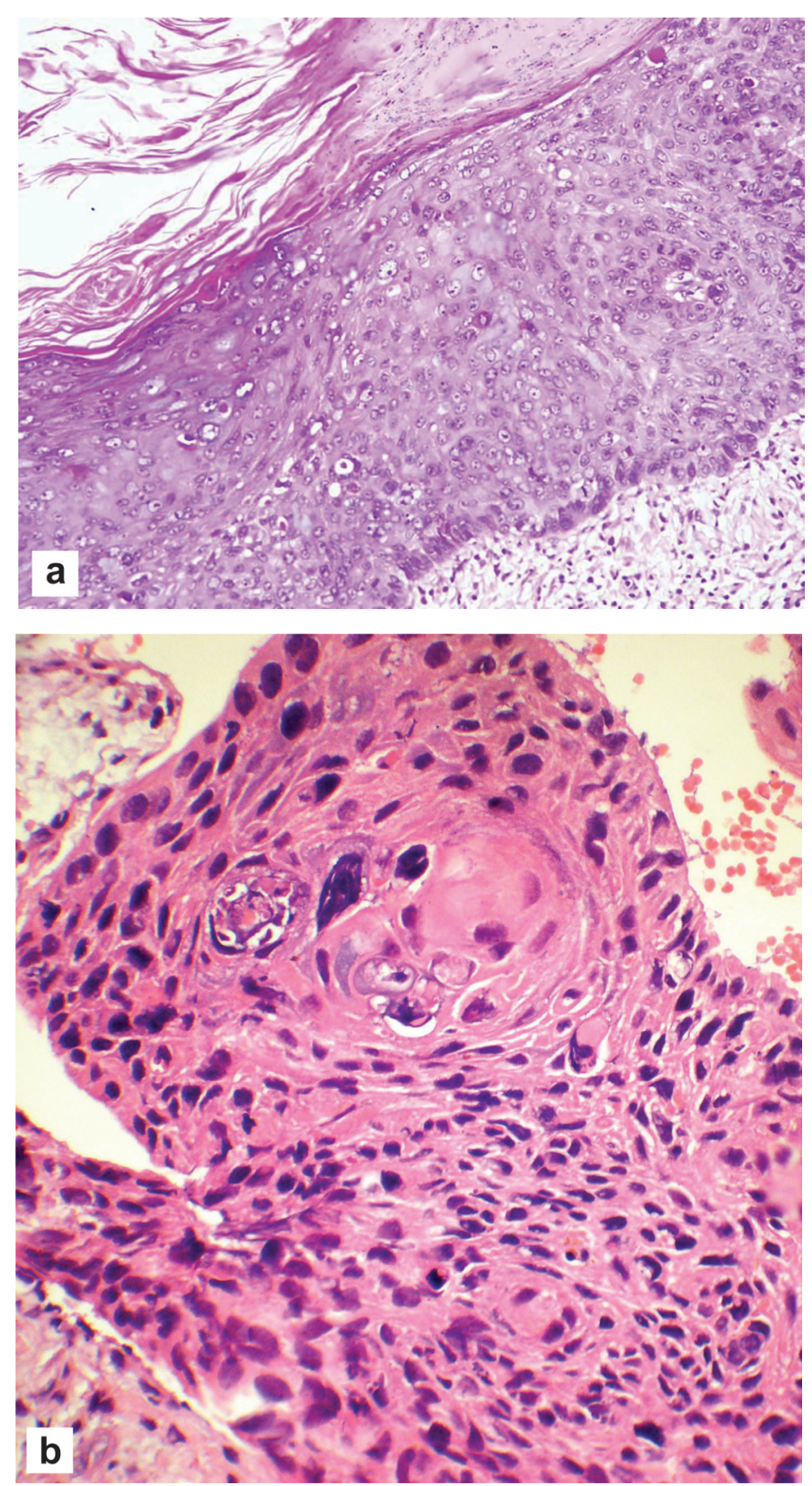

Figure 3 | (a) Focal full-thickness dysplasia (H\&E, 20x); (b) Bowen's disease showing hyperchromatic, pleomorphic nuclei, squamous pearl, and mitosis.

transplantation, in Hodgkin's disease and systemic lupus erythematosus, and with HIV infection (4). It is a multifactorial disease that involves genetic, immunological, and extrinsic (actinic) factors along with an unusual susceptibility to HPV infection. Recently, two adjacent novel genes EVER1 and EVER2 (Epidermodysplasia Verruciformis Enhancing Region) were identified. Mutations of these genes have been found only in EV and are said to be responsible for the development of the disease. These mutations have been recognized and reported in the EVER1/TMC6 or EVER2/ TMC8 (transmembrane channel-like protein 6 and 8) genes located on chromosome 17q25. Seven mutations in the EVER1 gene and five mutations in the EVER2 gene have been identified to date (2). EVER genes are transcribed in human skin keratinocytes, T lymphocytes, B lymphocytes, and NK cells, thereby impairing CMI and causing persistence of EV-associated HPV types and uncontrolled proliferation of stem cells and transient amplifying cells infected with $\mathrm{HPV}_{5}$ and HPV8, causing malignant transformation of some lesions $(1,2)$. Patel et al. have shown a link between the EVER2 gene and SCC with HPV5 and HPV8 as causative agents in $90 \%$ of cases (2). It has previously been shown that the potential 
of EV lesions for progression to cancer also depends on the HPV genotype detected in the lesion. HPV5, HPV8, HPV10, and HPV47 were found in more than $90 \%$ of EV-associated malignant neoplasms, whereas infection by HPV14, HPV20, HPV21, and HPV25 is usually associated only with the development of benign lesions (5). Multiple genome copies of HPV 5 and HPV8 have been detected in majority of cutaneous SCCs in patients with EV and have been classified as "possibly carcinogenic" in these patients. In addition to these, genome copies of HPV14, HPV20, and a few others have also been found (5). Because these HPVs persist extrachromosomally as free monomeric or oligomeric DNA molecules in a high copy number (100-300/diploid host genome), they undergo active transcription (6). This is in contrast to genital cancers, in which HPV DNA sequences are usually integrated into the host DNA (7). DNA amplification studies should be performed to identify specific HPV types, but due to financial constraints these studies could not be conducted for our patients (8).

Because the EV-HPV DNA sequences have only rarely been detected in premalignant and malignant skin lesions in the general population, this emphasizes the role of genetic and immunological factors in abnormal susceptibility to specific HPV types (7).

EV lesions generally appear in early childhood, as in all three of our cases; however, they may also start developing around middle age (4). With an average age of onset of 9 years, the disease manifests in either a benign or malignant form. The benign form is characterized by flat, wart-like, hypopigmented or hyperpigmented papules resembling tinea versicolor, whereas the malignant form presents as verrucous and seborrheic keratosis-like lesions on sun-exposed surfaces, including the face, hands, and feet $(6,9)$. At least $30 \mathrm{HPV}$ types have been characterized in benign EV lesions. EV has an unusual susceptibility to cutaneous beta HPV infection, which causes development of pityriasis versicolorlike lesions and flat warts. These beta HPVs that were identified

\section{References}

1. Emsen IM, Kabalar ME. Epidermodysplasia verruciformis: an early and unusual presentation. Can J Plast Surg. 2010;18:21-4.

2. Kalińska BA, Kowalewski C, Majewski S. The EVER genes-the genetic etiology of carcinogenesis in epidermodysplasia verruciformis and a possible role in non-epidermodysplasia verruciformis patients. Adv Dermatol Allergol. 2016;33:75-80.

3. Zahir A, Craig L, Rady P, Tyring S, Ehrlich A. Epidermodysplasia verruciformis associated with HPV 10. Dermatol Online J. 2013;19:2.

4. Gü U, Kılıç A, Gönül M, Çakmak SK, Bayis SS. Clinical aspects of epidermodysplasia verruciformis and review of the literature. Int J Dermatol. 2007;46:1069-72.

5. Howley PM, Pfister HJ. Beta genus papillomaviruses and skin cancer. Virology. 2015;479-480:290-6.

6. Quint KD, Genders RE, de Koning MN, Borgogna C, Gariglio M, Bouwes BC, et al. Human beta-papillomavirus infection and keratinocyte carcinomas. J Pathol. 2015;235:342-54.

7. Orth G. Epidermodysplasia verruciformis: model for understanding the oncogenity of human papillomaviruses. Ciba Found Symp. 1986;120:157-74. in warts, reddish-brown macular lesions, and cutaneous SCC of EV patients were initially referred to as EV-HPVs (5). Malignant transformation of a wart takes a period of about 2 to as many as 30 years in $35 \%$ to $50 \%$ of EV patients. Malignancies usually appear around age 40, but have been reported to occur as early as age 13 on areas exposed to the sun, such as the forehead and neck, as seen in Case 3. High-elevation and outdoor occupations hasten malignant conversion. A combination of chronic ultraviolet exposure and the possibility of EV-HPV in hair follicles may explain this malignant transformation in sun-exposed sites (10). Bowen'stype CIS is the most frequently encountered malignancy, followed by SCC, basal cell carcinoma, and rarely sweat gland carcinoma $(6,11)$. In EV patients, two deaths have been reported in the literature, one due to Burkitt's lymphoma and the other one due to disseminated reticulosis (12).

Treatment for EV includes preventive measures, especially strict sun avoidance and use of sunscreens as soon as the diagnosis is made. Systemic and topical retinoids, interferon, immunotherapy, electrodessication, and cryotherapy are the modalities tried for EV (1). Imiquimod stimulates cytotoxic T cell and B cell response (1). Acitretin (0.5-1 mg/ day) for 6 months is the palliative drug of choice (11). Topical imiquimod five times a week is very effective in the treatment of Bowen's disease and may be combined with systemic interferon therapy (6 million units, twice a week) in cases with multiple vivid presentations of HPV infections (13).

All of our patients were advised to strictly avoid sun exposure and to regularly use sunscreens and interferons. In addition, the patient with Bowen's disease was also advised to use topical imiquimod.

No therapy for EV is definitive; however, early diagnosis, sun protection, life-long observation for malignant transformation, and excision of the cancerous lesions are imperative for better survival (4).
8. Arora T, Sharma S, Sharma N, Titiyal IS. Bilateral recurrent ocular surface squamous cell cancer associated with epidermodysplasia verruciformis. BMJ Case Rep. 2015;2015.

9. Kambhampati SB, Vinay K, De D, Handa S, Gaspar BL, Saikia UN. Sebaceous cell carcinoma developing in epidermodysplasia verruciformis. Indian J Dermatol Venereol Leprol. 2016;82:433-5.

10. Nuovo GJ, Ishag M. The histologic spectrum of epidermodysplasia verruciformis. Am J Surg Pathol. 2000;24:1400-6.

11. Agrawal PG, Mahajan SA, Khopkar US, Kharka VD. Epidermodysplasia verruciformis: an unusual malignant transformation. Indian J Dermatol Venereol Leprol. 2013;79:97-9.

12. Lutzner MA. Epidermodysplasia verruciformis. An autosomal recessive disease characterized by viral warts and skin cancer. A model for viral oncogenesis. Bull Cancer. 1978;65:169-82.

13. Baskan EB, Tunali S, Adim SB, Turan A, Toker S. A case of epidermodysplasia verruciformis associated with squamous cell carcinoma and Bowen's disease: a therapeutic challenge. J Dermatolog Treat. 2006;17:179-83. 